\title{
VAC. 15 - Standardization of an in vitro assay for the evaluation of a candidate yellow fever vaccine
}

Andréa M. V. da Silva ${ }^{1 *}$; Tamiris Azamor ${ }^{2}$; Leonardo Ribeiro Batista-Silva ${ }^{1}$; Nathalia Suzano Villanova Benigno ${ }^{1}$; Thyago Leal Calvo ${ }^{3}$; Camilla Bayma ${ }^{1}$; Denise C S Matos ${ }^{1}$; Milton Ozorio Moraes ${ }^{3}$.

1Fiocruz/Bio-Manguinhos;

2Coordinación Nacional de Investigación, Universidad Privada Franz Tamayo - Bolívia; 3Fiocruz/IOC.

Introduction: Bio-Manguinhos is the world's biggest producer of the attenuated yellow fever vaccine (YFV17DD), a centennial product recognized as one of the most successful vaccines ever developed in terms of efficacy and safety. Besides, it is considered the standard vaccine to investigate the dynamics and quality of an ideal vaccine. Although, YFV17DD present rare cases of adverse events post-vaccination (AEPV) and Bio-Manguinhos has been seeking new strategies that achieve the necessary safety in AEPV risk groups. Although, production of new immunobiologicals is no longer empirical and has been improved by the use of technical-scientific approaches, which seek deeper interdisciplinary studies in different fields such as genetics and immunology. In this context, systems biology has been increasingly relevant and could help to design new vaccines.

Objective: To establish an analytical platform for gene expression in systems biology for in vitro evaluation of Yellow Fever candidate vaccines developed in Bio-Manguinhos using the YFV17DD vaccine as standard.

Methodology: Plates with 24 wells contained $1 \times 10^{6}$ cells of the monocytic human THP-1 cell line maintained in RPMI medium containing $10 \%$ fetal bovine serum. Cells were infected with attenuated YFV (AYFV) MOI 1, 0.002 and 0.001 and YFV17DD, or stimulated with 5, 10 and $20 \mu \mathrm{g} / \mathrm{mL}$ of envelope protein E of YFV (YFE) adjuvated or not, for 24, 48, 72 and 96 hours. Then, immunoenzymatic assay was used to viral and protein E quantitation. The genomic RNA was extracted and cDNA was synthesized followed by an RT-qPCR, to measure expression of the interferon pathway genes using RPL13 as house-keeping genes. From the supernatant were quantified the chemokines CCL2, CCL3, CXCL8, and CXCL10, using liquid microarray assay.

Results: It was observed that the THP1 model was susceptible to viral replication and stimulation with YFE, since YF RNA, and YFE protein are present until 96h. The expression of genes of IFN type I pathway was induced against the stimulus to all vaccines, however, presenting a higher induction with YFA. The incubation conditions that presented higher and more differentiative gene expression levels was the time of 48 hours and the MOI of 0,002 for YFA17DD and $5 \mu \mathrm{g} / \mathrm{mL}$ of the YFE. Our preliminary data also demonstrated that the stimulation with different yellow fever vaccines was able to induce increased of cytokines such as CCL2, CCL3, CXCL8, and CXCL10, in the monocyte cell model THP1.

Conclusion: All vaccines induced the expression of interferon pathway genes, inducing the production of chemokines by the monocyte cell line. Thus, the optimal culture parameters of the THP1 cells were determined, as well as the concentration of the vaccines used and the incubation time. The resuls contribute to the subsequent implementation of the analytical platform of genetic expression based on systems biology to be used as screening for evaluation of vaccines.

Keywords: In vitro model; yellow fever vaccine; systems biology 